\title{
Correlation of Protein Carbonyl and Protein Thiols in Oxidative Stress-induced Senescence of Red Blood Cell Membrane in Type 2 Diabetes Mellitus
}

\author{
${ }^{1}$ Veerabhadra Goud GK, ${ }^{2}$ Dinesh Javarappa
}

\begin{abstract}
Introduction: Diabetes mellitus (DM) is an endocrine dysfunction, which is associated with metabolic disease. Wherein chronic hyperglycemia presets the oxidative process which in turn biochemically leads to advanced glycation. The aim is to study the changes caused by oxidative stress upon the red blood cell (RBC) membrane composition by protein carbonyl and protein thiols in type 2 DM (T2DM) in comparison with that of controls.

Materials and methods: The study includes two groups, consists of 100 cases of T2DM and 100 healthy subjects as controls. Blood samples were collected under strict aseptic precautions as per the criteria and estimation of fasting blood sugar (FBS), hemoglobin $\mathrm{A} 1 \mathrm{c}(\mathrm{HbA} 1 \mathrm{c})$, protein carbonyl with $\mathrm{RBC}$ membrane ghost preparation and estimation of protein thiols were done.
\end{abstract}

Results: It was found that there was significant increase of protein carbonyl in cases $(1.20 \pm 0.08)$, in comparison to normal controls $(0.90 \pm 0.06)$ with a statistical significance of $(p<0.001)$ along with Protein thiols of RBC membrane which was significantly decreased $(1.42 \pm 0.10)$ in cases in comparison to normal controls. $(2.12 \pm 0.12)$ with a statistical significance of $(p<0.001)$. In our study, the positive correlation of protein carbonyl and protein thiols was established with $74 \%$ of cases of type $2 \mathrm{DM}$ falling into a $\mathrm{HbA} 1 \mathrm{C}$ control group of 7 to $8 \%$ indicating the increased protein carbonyl and decreased protein thiols are early indicators of progressive diabetic changes.

Conclusion: Changes in RBC membrane of persons with T2DM acts as an early indicator by means of an increase in protein carbonyl and decrease in protein thiols, and this will further lead to progressive diabetic changes.

Keywords: Diabetes mellitus (DM), Oxidative stress, Protein carbonyl, Protein thiols, Reactive oxygen species.

How to cite this article: Goud GKV, Javarappa D. Correlation of Protein Carbonyl and Protein Thiols in Oxidative Stress-induced Senescence of Red Blood Cell Membrane in Type 2 Diabetes Mellitus. Indian J Med Biochem 2018;22(2):143-146.

Source of support: Nil

Conflict of interest: None

${ }^{1}$ Associate Professor, ${ }^{2}$ Professor and Head

${ }^{1}$ Department of Biochemistry, Akash Institute of Medical Sciences and Research Centre, Bengaluru, Karnataka, India

${ }^{2}$ Department of Biochemistry, Vishwbharathi Medical College and General Hospital, Penchikalapadu, Kurnool, Andhra Pradesh, India

Corresponding Author: Dinesh Javarappa, Professor and Head, Department of Biochemistry, Vishwbharathi Medical College and General Hospital, Penchikalapadu, Kurnool, Andhra Pradesh, India, Mobile: +919845636830, e-mail: drdineshjavarappa@ gmail.com

\section{INTRODUCTION}

Diabetes is a significant health problem affecting people all over the world. In India, T2DM may increase from 50.8 million in 2010 to 87.0 million by 2030 . The DM is a being a metabolic disease associated with chronic hyperglycemia and relative lack of insulin. Chronic hyperglycemia leads to damage and dysfunction of various organ systemseyes, kidneys, nerves system, blood vessels, and heart.

Diabetes mellitus (DM) is a metabolic disease characterized by a state of chronic hyperglycemia resulting from defects in Insulin secretion, Insulin action or both. The chronic hyperglycemia is associated with long-term damage, dysfunction, and failure of normal functioning of various organs, especially the eyes, kidneys, nerves, heart, and blood vessels. Chronic hyperglycemia leads to the generation of free radicals, especially reactive oxygen species (ROS) from glucose autooxidation and protein glycation. The teratogenic and carcinogenic effects of oxidative stress are because of modification in protein, lipid, and deoxyribonucleic acid (DNA), which ultimately causes cellular dysfunction. ${ }^{1}$

During DM, oxidative stress will lead to various complications. Increased levels of ROS in DM is because of their increased production and/or decreased destruction by antioxidants such as non-enzymatic or enzymatic reactions like catalase, reduced glutathione (GSH) and superoxide dismutase (SOD). ${ }^{2}$ Oxidative stress is currently suggested as the mechanism underlying DM and its complications. ${ }^{3}$ Oxidative stress leads to proteins, lipids and DNA modifications that cause cellular dysfunction and this could have teratogenic or carcinogenic consequences. ${ }^{4}$ Over the last few decades, several age-related alterations of erythrocytes have been investigated), ${ }^{5}$ the oxidative damage, initiated by ROS and other oxidants, upon the alteration of erythrocytes has been investigated. ${ }^{5}$ In case of thalassemia, oxidative damage to the erythrocyte membrane plays an essential role in the aging (senescence) of pathological red cells, ${ }^{6}$ of these oxidative damages to the erythrocyte membrane component is presently thought to play a pivotal role during the senescence of pathological red cells in thalassemia, sickle cell disease, etc.

A characteristic hallmark of many pathophysiological conditions is a decrease in the GSH: oxidized glutathione 
(GSSH) ratio, The GSSG accumulates in the cells, it can undergo disulfide exchange reaction with protein thiols leading to S-glutathionylation. These S-glutathionylated protein has been investigated as a positive biomarker of oxidative stress in human disease such as DM.

In most of the pathological conditions, the accumulation of protein carbonyls has been observed. Protein carbonyl is the most commonly used marker and is also the most common indicator of protein oxidation. There is an appearance of carbonyl groups such as aldehyde/ ketone groups of proteins which are caused because of oxidative modification. Protein carbonyls are more hydrophobic, and they resist proteolysis. ${ }^{4}$ The study was taken up in both normal subjects as well as those with T2DM to assess the effects of oxidative stress on the erythrocyte membrane.

\section{MATERIALS AND METHODS}

The study was conducted in Basaveshwara Medical College and Hospital, Chitradurga, a tertiary care center. After doing the biochemical tests of $\mathrm{HbA1}$ c in our central laboratory, 100 individuals with $\mathrm{HbA} 1 \mathrm{c}$ levels more than $7 \%$ were taken as T2DM. One-hundred individuals with age and sex-matched healthy individuals with $\mathrm{HbA1c}$ $<5.3 \%$ taken as controls. Both cases and control subjects were subjected to estimation of biochemical parameters. Venous blood samples were collected after taking aseptic precautions from the study subjects. Five $\mathrm{mL}$ of blood was collected in plain vacuum tubes, and $2 \mathrm{~mL}$ of blood was collected in ethylenediaminetetraacetic acid (EDTA) vacuum tubes. Samples were left for 20 minutes at room temperature and centrifuged at $3000 \mathrm{rpm}$ for 4 to 5 minutes. Serum was used for the estimation of FBS by glucose oxidase (GOD)-peroxidase (POD) method.? Estimation of HBA1C was done by cation exchange resin method. ${ }^{8}$ RBC membranes were prepared by Dodge et al. method, ${ }^{9}$ protein carbonyl estimation was done by Levine et al. method ${ }^{10}$ and protein thiols was estimated by Habeeb AFSA, methods enzymol. ${ }^{11}$ The study was approved by the ethics committee, a written informed consent was obtained from all the participants in this study.

Table 1: Comparison of FBS, HbA1c, protein carbonyl and protein thiols between type $2 \mathrm{DM}$ patients and healthy controls

\begin{tabular}{llll}
\hline Parameters & Cases $(n=100)$ mean $\pm S D$ & Controls $(n=100)$ mean $\pm S D$ & $p$-value \\
\hline Age & $41.52 \pm 5.47$ & $41.52 \pm 5.47$ & $<0.001^{* *}$ \\
Fasting blood sugar $(\mathrm{mg} / \mathrm{dL})$ & $197.50 \pm 62.84$ & $93.48 \pm 7.54$ & $<0.001^{* *}$ \\
HbA1C (\%) & $7.73 \pm 0.67$ & $5.34 \pm 0.59$ & $<0.001^{* *}$ \\
Protein Carbonyl (nmoles/mg of protein) & $1.20 \pm 0.08$ & $0.90 \pm 0.06$ & $<0.001^{* *}$ \\
Protein Thiols (nmoles/mg of protein) & $1.42 \pm 0.10$ & $2.12 \pm 0.12$ & $<\mathrm{s} 0.001^{* *}$ \\
\hline
\end{tabular}

** Statistically Significant 
This exposure of the protein to oxygen free radicals results in protein damage, a process of oxidative modification of many amino acid residues, fragmentation, aggregation, and increased protein susceptibility. They not only limit to protein but further cause damage to lipids and nucleic acids as well as leading to various diabetic complications ${ }^{13}$ over a due course of time.

Biological systems are continually exposed to endogenous and exogenous ROS, and RNS and proteins are their major targets due to abundance, relatively long half-lives and high rate of reactions. However, the overall biology of oxido-nitrosative protein modifications remains complex and is not completely defined, but protein carbonylation is quite well characterized. Protein carbonylation is an irreversible, non-enzymatic modification of proteins. The carbonyl groups are introduced into proteins by a variety of oxidative/nitrosative pathways. ROS and RNS can react directly with the protein and generating products such as reactive carbonyl species (RCS) then react with the proteins.

Direct oxidation of proteins by ROS and RNS yields highly RCS derivatives resulting from oxidation of lysine, arginine, proline, and threonine amino acid residues. Carbonyl groups can also be generated by the reaction of the primary amino group of lysine with RCS such as ketoamines, ketoaldehydes, deoxyosones and eventually leading to the formation of advanced glycation end products (AGEs). Now it is well accepted that oxidation/nitration of proteins typically results in an increase in carbonyl contents. This increase is due to the oxido-nitration of lysine, arginine, proline and threonine residues. In short, protein carbonyl groups are the most reliable biomarker of oxidative/nitrosative stress. ${ }^{14}$

Cellular proteins are believed to be the targets of free radicals induced oxidation injury. Protein carbonyl content in the cells is one indication of oxidative damage to proteins and can be generated via. nonspecific oxidation of amino acids or catalyzed oxidation of specified amino acid key to protein function by oxygen and glycation. Protein carbonyl is generated by oxidative modifications of proteins either by alpha-amidation ( $\alpha$-amidation) pathway or by oxidation of glutamyl side chains, reactions with aldehydes like malondialdehyde produced during lipid peroxidation, oxidation of reducing sugars and reaction of oxidized product with lysine residues of proteins. ${ }^{15}$

Sagare et al., did a study on T2DM and its complications, by recruiting 50 T2DM cases and 50 age and sexmatched healthy subjects. They estimated protein carbonyl, glycated hemoglobin ( $\mathrm{HbA1c}$ ) and urine microalbumin. They found that there is a significantly increased level of protein carbonyls, $\mathrm{HbA} 1 \mathrm{c}$, and urine microalbumin in
T2DM cases when compared with controls. Our study results are also in accordance with these findings. ${ }^{16}$

In the present study, protein thiols content of RBC membrane was significantly decreased in cases when compared to controls, which exhibits the free radical injury due to the sulfhydryl groups and protein mixed disulfides with homocysteine and glutathione are found as low molecular weight disulfide pool in reduced form, which results in oxidation of cysteine residues and reversible formation of mixed disulfides among low molecular mass thiols (GSH), which can directly alter the orb regulate protein function for (redox function) and can have a role in protein oxidation, resulting in senescence of $R B C$ membrane. ${ }^{17,18}$

\section{CONCLUSION}

Our study revealed that there was an increase in protein carbonyl content in cases as compared with that of controls. One of the indications for oxidative damage is the presence of protein carbonyl content in the plasma membrane of erythrocytes. Oxidative damage leads to modification of proteins by oxidation of glutamyl side chains or by $\alpha$-amidation pathway, reaction with malondialdehyde, which is produced during reaction with that of oxidized products with lysine residue of proteins oxidation of reducing sugars and lipid peroxidation. Further studies have to be done to diagnose early changes in type 2 diabetics to identify high-risk subjects and to prevent further complications.

\section{REFERENCES}

1. Tiwari BK, Pandey KB, Abidi AB, Rizvi SI. Markers of Oxidative Stress during Diabetes Mellitus. Journal of Biomarkers [Internet]. Hindawi Limited; 2013;2013:1-8.

2. Kangralkar VA, Patil SD, Bandivadekar RM. Oxidative stress and Diabetes: A Review. Int. J. Pharma Applications. 2010;1: 38-45.

3. Mousa SA, Oxidative stress in DMs. Roman J Biophys. 2008;18(3);222-236.

4. Kalaivanam KN, Kumar SN, Bheemasen R, Mahendran BK. Role of protein oxidation and lipid peroxidation markers status in NIDDM patients. World Journal of Pharmacy and Pharmaceutical Sciences. 2014;3(6 ):1510-1520.

5. Fujino T, Ando K, Beppu M, Kikkugawa K. Enzymatic removal of protein aggregates from erythrocyte membranes. J.Biochem. 2000;127(6):1081-1086.

6. Celedone G, Rodriguez I, Espana J, Lissi E. Contribution of Hemoglobin and membrane constituents modification to human erythrocyte damage produced by peroxyradicals of different charge and hydrophobicity. Free Radic. Res 2001;34:17-31.

7. Burtis CA. Carbohydrates in Sacks DD ednt. Clinical chemistry. Chapter 22. 6th edition. Saunders 2008;390-391.

8. Gowenlock AH, McMurray JR, McLauchen DM, Tests in disorders of Glucose metabolism. In: Weiner K edn. Practical 
clinical Biochemistry. Chapter 25. 6th edition. CBS Publishers. 1996:333-349.

9. Dodge JT, Mitchell C. Hanahan DJ, The preparation and chemical characteristics of Hemoglobin free ghosts of human erythrocyte. Arch Biochem Biophys. 1963:100119-100213.

10. Levine RL, William JA, Stadtman ER, Shacter E. Carbonyl assays for determination of oxidativley modified proteins, Methods Enzymol. 1994;233:346-357.

11. Habeeb AF. Reaction of protein sulfhydryl groups with Ellman's reagent. Reaction of protein sulfhydryl groups with Ellman's reagent. Methods Enzymol. 1972;25:457-464.

12. Stryer L. Portrait of an allosteric protein. Biochemistry. 1995:154.

13. Mallick AK, Maradi R, Joshi VR, Shorey G, Ahsan M. Study on malondialdehyde as a marker of lipid peroxidation in male and female patients with type 2 diabetes mellitus. International Journal of Pharmaceutical Sciences Review and Research. 2011;8(2):198-201.
14. Almogbel E, Rasheed N. Protein Mediated Oxidative Stress in Patients with Diabetes and its Associated Neuropathy: Correlation with Protein Carbonylation and Disease Activity Markers. Journal of clinical and diagnostic research: JCDR. 2017 Feb;11(2):BC21-BC25.

15. Deokar P, Jagtap A, Yerawar C. Correlation of protein carbonyl and MDA in diabetes and its complications. Indian Journal of Basic and Applied Medical Research. 2016;5(2):284-289..

16. Sagare AA, Trivedi DJ, Patil VS, Kulkarni SP. Protein Carbonyl \& Microalbuminuria in Type 2 Diabetes Mellitus. Indian Journal of Basic and Applied Medical Research; 2012 Dec;2(5):399-404.

17. Gabreanu GR, Angelescu S. Erythrocyte membrane in type 2 diabetes mellitus. Discoveries [Internet]. Applied Systems, srl; 2016 Jun 30;4(2):e60.

18. Lowry OH, Rosebrough NJ, Farr AL, Randall RJ. Protein measurment with the Folin phenol reagent. J Biol Chem. 1951. Nov;193(1):265-275. 